\title{
ORBITS IN FINITE INCIDENCE STRUCTURES
}

\section{INTRODUCTION}

A finite incidence structure $(S, X, \mathrm{I})$ consists of finite sets $S$ and $X$, the points and blocks of the structure, where the matrix I defines the incidence of a point with a block. In this paper we consider tactical decompositions of a structure into point classes and block classes. The interest in tactical decompositions stems from the fact that they are generalizations of the orbit partitions under the action of a collineation group. Therefore the results in this paper apply in particular to groups of automorphisms of a finite structure.

Let $P_{S}$ be an arbitrary partition of $S$ and $P_{X}$ an arbitrary partition of $X$. In Theorem 2.3 we construct a refinement $\left(P_{S}^{*}, P_{X}^{*}\right)$ which is a tactical decomposition of the structure. We show that $\left(P_{S}^{*}, P_{X}^{*}\right)$ is unique with respect to the minimality of the number of classes in $P_{S}^{*}$ and $P_{X}^{*}$. The algorithm that produces this tactical decomposition only uses some elementary linear algebra and does not involve automorphisms of the incidence structure. This suggests that tactical decompositions might be of particular interest for the investigation of rigid structures admitting only the identity collineation.

In Section 3 we consider structures in which the point cardinality is equal to the rank of I. Here we prove, that for a tactical decomposition the block partition entirely determines the point classes. Our result (Theorem 3.1) contains a number of well-known theorems. If the structure under consideration is a 2-design, for instance, we obtain a generalization and an independent proof of the Dembowski-Parker-Hughes theorem. In the case of the subset lattice of a finite set the theorem improves a now classical result by Livingstone and Wagner. A similar orbit theorem is obtained for the subspace lattice of finite affine and projective geometries. In a forthcoming paper [13] I have proved that certain general classes of finite graphs have maximal incidence rank. Here we obtain yet another orbit theorem.

In Section 4, finally, we investigate the relationship between the point action and the block action of an automorphism of the structure. Here we show that again the block permutation completely determines the action on points if the rank of $I$ is the cardinality of $S$. In Theorem 4.2 we give a formula for the number of point orbits of given length in terms of the block permutation.

\section{TACTICAL DECOMPOSITIONS}

Let $S$ and $X$ be finite sets and I a $(0,1)$-incidence matrix where the rows of $I$ are indexed by $S$ and the columns by $X$. We call the elements of $S$ points and 
the elements of $X$ blocks. We say 's is incident with $x$ ', and denote this by $s \mathrm{I} x$, in the case that the $(s, x)$-entry of $\mathrm{I}$ is 1 . The triple $(S, X, \mathrm{I})$ is called a finite incidence structure.

For a field $R$ of characteristic zero we define the space $R S$ as the vector space over $R$ whose basis vectors are the points in $S$. In a similar way we define the block space $R X$. Two linear maps arise naturally from the incidence relation: $\partial^{-}: R X \rightarrow R S$, given by $\partial^{-}(x)=\Sigma_{s I x} s$ for all $x$ in $X$ and $\partial^{+}: R S \rightarrow R X$, given by $\partial^{+}(s)=\Sigma_{s i x} x$ for all $s \in S$. The matrices of $\partial^{-}$and $\partial^{+}$(for the bases $S$ and $X$ ) are, of course, the incidence matrix I and its transpose $I^{t}$, respectively.

Let $P_{S}$ be a partition of $S$ into disjoint classes $P_{S, 1}, \ldots, P_{S, i}, \ldots$ and define the vector $p_{i}$ in $R S$ as the sum over all points in the class $P_{S, i}$. As $P_{S}$ is a partition, the $p_{i}$ 's are linearly independent. Hence they form a basis of the subspace $R P_{S}=\left\langle p_{i} \mid i=1, \ldots\right\rangle$ of $R S$ which corresponds to the partition $P_{S}$. Vice versa, we call a subspace of $R S$ a partition space, provided it has a basis $p_{1}, \ldots, p_{i}, \ldots$ such that the $p_{i}$ 's have the above form for some partition of $S$. It is crucial for our argument and easily verifiable that partitions and partition spaces are in one-one correspondence so that $P_{S}$ is uniquely determined by $R P_{S}$. The partial order relation between two partitions $P_{S}$ and $P_{S}^{\prime}$ of the point set is given by $P_{S} \leqslant P_{S}^{\prime}$ if and only if every class of $P_{S}^{\prime}$ is a subclass of some class in $P_{S}$. We say $P_{S}^{\prime}$ is finer than or equal to $P_{S}$ in this case. Clearly $P_{S} \leqslant P_{S}^{\prime}$ is equivalent to $R P_{S} \subseteq R P_{S}^{\prime}$.

For a partition $P_{X}$ of the block set into disjoint classes $P_{X, 1}, \ldots, P_{X, j}, \ldots$ the vectors $q_{j}=\Sigma_{x \in P_{X, j}} x$ and the partition space $R P_{X}=\left\langle q_{j} \mid j=1, \ldots\right\rangle \subseteq$ $R X$ are defined in the same fashion. In the following we consider pairs $\left(P_{S}, P_{X}\right)$ of partitions of $S$ and $X$ respectively.

DEFINITION. A pair $\left(P_{S}, P_{X}\right)$ is a tactical decomposition of the incidence structure $(S, X, \mathrm{I})$ provided the following two dual conditions are satisfied:

$\mathrm{T}^{-}$: For any given point $s$ and any $j$, the number of blocks in $P_{X, j}$ incident with $s$ only depends on the class $P_{S, i}$ that contains $s$.

$\mathrm{T}^{+}$: For any given block $x$ and any $i$, the number of points in $P_{S, i}$ incident with $x$ only depends on the class $P_{X, j}$ that contains $x$.

Tactical decompositions (for which also the term generalized orbits is in common use) were first investigated by Dembowski [5] and are characterized by the following invariance conditions:

PROPOSITION 2.1. The pair $\left(P_{S}, P_{X}\right)$ satisfies $\mathrm{T}^{-}$if and only if $\partial^{-}\left(R P_{X}\right)$ is a subspace of $R P_{S}$. Similarly, $\left(P_{S}, P_{X}\right)$ satisfies $\mathrm{T}^{+}$if and only if $\partial^{+}\left(R P_{S}\right)$ is contained in $R P_{X}$.

Proof. Let $q=\Sigma_{x \in P_{X}, j} x$ be a basis vector of $R P_{X}$ and $\partial^{-}(q)=\Sigma_{x \in P_{X, j}} \Sigma_{s I x} s=$ $v$. We arrange this sum into $v=\Sigma_{s \in S} v_{s} \cdot s=\Sigma_{i} \Sigma_{s \in P_{s, i}} v_{s} \cdot s,\left(v_{s} \in R\right)$, using the fact that $P_{S}$ is a partition of $S$. We observe that $v$ is contained in $R P_{S}$ if and 
only if $v_{s}=v_{s^{\prime}}$ whenever $s$ and $s^{\prime}$ belong to the same class. This, on the other hand, is equivalent to the condition $\mathrm{T}^{-}$. The second part of the proof is just the dual of the first.

We will use the proposition to calculate tactical decompositions. First, however, we need the concept of the point partition generated by a subset $W$ of $R S$.

Let $v=\Sigma_{s \in S} v_{s} \cdot s$ be any vector in $W$. We write $v$ uniquely as $\Sigma_{i} r_{i} \cdot \Sigma_{k} s_{i k}$ in such a way that each point in $S$ occurs exactly once among the $s_{i k}$ and $r_{i} \neq r_{i}$ if $i \neq i^{\prime}$. Thus $v$ produces a partition $P_{S}(v)$ of $S$ with classes $\left\{s_{1, k} \mid k=\right.$ $1, \ldots\}, \ldots,\left\{s_{i, k} \mid k=1, \ldots\right\}$ and so on. If we interpret $v$ as an $R$-valued function on $S$, the classes of $P_{S}(v)$ are precisely the level surfaces of $v$, i.e. the point sets where $v$ has constant value $r_{i}$ (compare also to chapter 10 in Wielandt [14]).

Now let $P_{S}(W)$ be the coarsest partition of $S$ for which $P_{S}(v) \leqslant P_{S}(W)$ for all $v \in W$. Let $L(W)$ denote the partition space $R P_{S}(W)$. The following is an immediate consequence of these definitions.

PROPOSITION 2.2. Let $W \subseteq R S$ and $R P_{S}$ some partition space containing $W$. Then $W \subseteq L(W) \subseteq R P_{S}$ and $W=L(W)$ if and only if $W$ is a partition space itself.

Subspaces $U$ of $R X$ define partitions of $X$ and partition spaces $L(U)$ in the same way. We can now state the main result of this section.

THEOREM 2.3. Let $(S, X, \mathrm{I})$ be a finite incidence structure and let $P_{S}$ and $P_{X}$ be partitions of the points and blocks, respectively. Then there is a unique tactical decomposition $\left(P_{S}^{*}, P_{X}^{*}\right)$ with minimal class numbers satisfying $P_{S} \leqslant$ $P_{S}^{*} \leqslant P_{S}^{\prime}$ and $P_{X} \leqslant P_{X}^{*} \leqslant P_{X}^{\prime}$ for any tactical decomposition $\left(P_{S}^{\prime}, P_{X}^{\prime}\right)$ with $P_{X} \leqslant P_{S}^{\prime}$ and $P_{X} \leqslant P_{X}^{\prime}$.

Proof. The tactical decomposition $\left(P_{S}^{*}, P_{X}^{*}\right)$ can be constructed in the following fashion. Since $P_{S} \leqslant P_{S}^{\prime}$ and $P_{X} \leqslant P_{X}^{\prime}$, we obtain $R P_{S} \subseteq \partial^{-}\left(R P_{X}\right)+$ $R P_{S} \subseteq R P_{S}^{\prime}$ and $R P_{X} \subseteq \partial^{+}\left(R P_{S}\right)+R P_{X} \subseteq R P_{X}^{\prime}$ by Proposition 2.1, as $\left(P_{S}^{\prime}, P_{X}^{\prime}\right)$ is a tactical decomposition. By Proposition 2.2, we also have $R P_{S} \subseteq$ $L\left(\partial^{-}\left(R P_{X}\right)+R P_{S}\right) \subseteq R P_{S}^{\prime}$ and $R P_{X} \subseteq L\left(\partial^{+}\left(R P_{S}\right)+R P_{X}\right) \subseteq R P_{X}^{\prime}$. We now define $P_{S}^{1}$ by $R P_{S}^{1}=L\left(\partial^{-}\left(R P_{X}\right)+R P_{S}\right)$ and $P_{X}^{1}$ by $R P_{X}^{1}=L\left(\partial^{+}\left(R P_{S}\right)+R P_{X}\right)$. Thus we obtain $R P_{S} \subseteq R P_{S}^{1} \subseteq L\left(\partial^{-}\left(R P_{X}^{1}\right)+R P_{S}^{1}\right) \subseteq R P_{S}^{\prime}$ and $R P_{X} \subseteq R P_{X}^{1} \subseteq$ $L\left(\partial^{+}\left(R P_{S}^{1}\right)+R P_{X}^{1}\right) \subseteq R P_{X}^{\prime}$ as above. Inductively, let $P_{S}^{i}$ and $P_{X}^{i}$ be given by $R P_{S}^{i}=L\left(\partial^{-}\left(R P_{X}^{i-1}\right)+R P_{S}^{i-1}\right)$ and $R P_{X}^{i}=L\left(\partial^{+}\left(R P_{S}^{i-1}\right)+R P_{X}^{i-1}\right)$, respectively. In this way, we obtain two ascending chains $R P_{S} \subseteq R P_{S}^{1} \subseteq \cdots \subseteq R P_{S}^{i} \subseteq$ $\cdots \subseteq R P_{S}^{\prime}$ and $R P_{X} \subseteq R P_{X}^{1} \subseteq \cdots \subseteq R P_{X}^{i} \subseteq \cdots \subseteq R P_{X}^{\prime}$ which become stationary as all dimensions are finite. Thus, for $R P_{S}^{k}=R P_{S}^{k+1}$ and $R P_{X}^{k}=R P_{X}^{k+1}$ we conclude $\partial^{-}\left(R P_{X}^{k}\right) \subseteq R P_{S}^{k}$ and $\partial^{+}\left(R P_{S}^{k}\right) \subseteq R P_{X}^{k}$. Hence, according to Proposition $2.1, P_{S}^{*}=P_{S}^{k}$ and $P_{X}^{*}=P_{X}^{k}$ are the partitions of a tactical decomposi- 
tion. Uniqueness now easily follows from the fact that $P_{S}^{*} \leqslant P_{S}^{\prime}$ and $P_{X}^{*} \leqslant P_{X}^{\prime}$ hold for an arbitrary tactical decomposition finer than or equal to $\left(P_{S}, P_{X}\right)$. This completes the proof.

\section{ORBIT THEOREMS}

For any incidence structure, the orbits on points and blocks of an automorphism group $G$ form a tactical decomposition. We denote point orbits by $S(G)$ and block orbits by $X(G)$, where $G$ is an arbitrary group of automorphisms of the structure. Clearly the partitions $P_{S}=\{S\}$ and $P_{X}=\{X\}$ (consisting of one class each) satisfy $P_{S} \leqslant S(G)$ and $P_{X} \leqslant X(G)$. According to Theorem 2.3 , the tactical refinement $\left(P_{S}^{*}, P_{X}^{*}\right)$ of $\left(P_{S}, P_{X}\right)$ has the property $P_{S}^{*} \leqslant S(G)$ and $P_{X}^{*} \leqslant X(G)$ for any group of automorphisms. I wish to emphasize at this point that this minimal tactical decomposition is obtained quite independently of automorphisms. Therefore $\left(P_{S}^{*}, P_{X}^{*}\right)$ and, in particular, their class numbers are invariants related to the symmetries of an incidence structure which so far have not been examined. The class number $\left|P_{S}^{*}\right|$, for instance, is a lower bound for the number of point orbits of all automorphisms. In general, however, one cannot conclude that this bound is attained by any automorphism group: in many instances tactical decompositions cannot be associated to automorphisms. This fact has already been pointed out in Dembowski's paper [5] in the case of projective planes.

In this section we consider a given tactical decomposition of a finite structure and study the relationship between its point and orbit partitions. In many geometrical situations the number of point orbits of an automorphism group is known to be less than or equal to the number of block orbits. The following theorem shows that much more can be proved.

THEOREM 3.1. Let $(S, X, \mathrm{I})$ be a finite incidence structure and assume that the rank of $\mathrm{I}$ is equal to the number of points in $S$. If $\left(P_{S}, P_{X}\right)$ is a tactical decomposition, then the block partition $P_{X}$ uniquely determines the point partition through the relation $\partial^{-}\left(R P_{X}\right)=R P_{S}$. Moreover, the number of classes in $P_{S}$ is given by $\left|P_{S}\right|=\left|P_{X}\right|-\operatorname{dim}\left(\left(\operatorname{kern} \partial^{-}\right) \cap R P_{X}\right)$.

Proof. By the assumption on the rank of $\mathrm{I}$, the map $\partial^{+}: R S \rightarrow R X$ is injective. Since $\partial^{-}$and $\partial^{+}$are adjoint to each other in the standard inner products, $\partial^{-} \partial^{+}$is injective, and hence is a bijection on $R S$. If $\left(P_{S}, P_{X}\right)$ is a tactical decomposition, by Proposition 2.1, the map

$$
\partial^{-} \partial^{+}: R P_{S} \stackrel{\partial^{+}}{\longrightarrow} R P_{X} \stackrel{\hat{o}^{-}}{\longrightarrow} R P_{S}
$$

is also a bijection. This implies $\partial^{-}\left(R P_{X}\right)=R P_{S}$.

Hence the point partition is determined by the block partition. Since the class numbers are the dimensions of $R P_{S}$ and $R P_{X^{\prime}}$ we get $\left|P_{S}\right|=\left|P_{X}\right|-$ $\operatorname{dim}\left(\left(\operatorname{kern} \partial^{-}\right) \cap R P_{X}\right)$. This completes the proof. 
In case that the rank of $I$ is not maximal, the point partition is, in general, only partially determined by $L\left(\partial^{-}\left(R P_{X}\right)\right) \subseteq R P_{S}$. The argument here can easily be modified to show that $\left|P_{S}\right| \leqslant\left|P_{X}\right|-\operatorname{dim}\left(\left(\operatorname{kern} \partial^{-}\right) \cap R P_{X}\right)+|S|-$ rank I. (This is the content of Theorem 2.1 in Block [2].) The following list, however, shows that the condition on the maximality of the rank of $I$ is quite general.

1 . Let $S$ be the class of $k$-element subsets and $X$ the class of $l$-element subsets of a set of size $n$, where $k \leqslant l$ and $k+l \leqslant n$. If incidence is defined by set inclusion, the rank of $I$ is $|S|$. In this case Theorem 3.1 generalizes the theorem of Livingstone and Wagner. For reference compare [3], [9] and [13].

2. The incidence matrix of a 2-design has point rank. This is the essence of Fisher's inequality. Here the second part of Theorem 3.1 contains the Dembowski-Parker-Hughes theorem in [5], [7] and [10].

3. Affine and projectice spaces: Consider the $d$-dimensional affine and projective spaces $A G(d, q)$ and $P G(d, q)$ over the field of $q$ elements. Let $S$ be the collection of $e$-dimensional and $X$ the collection of $f$-dimensional subspaces where the incidence relation is inclusion. Kantor [8] has shown that I has maximal rank, and hence $\operatorname{rank} \mathrm{I}=|S|$ if $0 \leqslant e<f \leqslant d-e-1$.

4. Graphs: Let $S$ be the vertices and $X$ the edges of a finite undirected graph without loop edges. The incidence matrix of such a graph has point rank if and only if each component contains some cycle of odd length. This result is proved in a forthcoming paper [13]. Here Theorem 3.1 is an orbit theorem for graphs.

The point partition of a tactical decomposition does not, in general, determine the block partition. A structure, for instance, may have two automorphism groups which are both point transitive, but which have non-identical orbits on blocks. Some information about $P_{X}$, however, can be ascertained from the fact that $L\left(\partial^{+}\left(R P_{S}\right)\right) \subseteq R P_{X}$. Thus, if $P_{X}^{*}$ is the partition belonging to $L\left(\partial^{+}\left(R P_{S}\right)\right)$, then $P_{X}^{*} \leqslant P_{X}$, regardless of the rank of I.

\section{AUTOMORPHISMS}

An automorphism of a structure consists of a permutation $g_{S}$ of $S$ and a permutation $g_{X}$ of $X$ such that incidence is preserved: $s I x$ if and only if $g_{S}(s) I g_{X}(x)$. These permutations extent to linear maps on $R S$ and $R X$. One verifies easily that $g=\left(g_{S}, g_{X}\right)$ is an automorphism if and only if $\partial^{+} g_{S}=$ $g_{X} \partial^{+}$or, equivalently, $\partial^{--} g_{X}=g_{S} \partial^{-}$. For convenience the corresponding permutation matrices will also be denoted by $g_{S}$ and $g_{X}$.

THEOREM 4.1. Let $(S, X, \mathrm{I})$ be a finite incidence structure with rank $\mathrm{I}=|S|$ and let $\left(g_{S}, g_{X}\right)$ be an automorphism of the structure. Then $g_{X}$ uniquely determines $g_{S}$. If $g_{X}$ is an arbitrary permutation of $X$ then $g_{X}$ is the block permuta- 
tion of an automorphism if and only if $\partial^{-} g_{X} \partial$ is a permutation of $S$ independent of the choice of $\partial$ as a right inverse of $\partial^{-}$.

Proof. Since rank $\partial^{-}=\operatorname{rank} I=|S|$ by assumption, $\partial^{-}$has a right inverse. If $\left(g_{S}, g_{X}\right)$ is an automorphism, $\partial^{-} g_{X}=g_{S} \partial^{-}$implies $\partial^{-} g_{X} \partial=g_{S}$ for any right inverse $\partial$ of $\partial^{-}$. Hence $g_{X}$ determines $g_{S}$. If $g_{X}$ is an arbitrary permutation for which $\partial^{-} g_{X} \partial=g_{S}$ is a permutation independent of $\partial$, we have to show that $\partial^{-} g_{X}=g_{S} \partial^{-}$. Let $v$ be the difference between two right inverses of $\partial^{-}$. Then $\partial^{-} v=0$. By assumption we have $\partial^{-} g_{X} v=0$. Taking $v=\left(\mathrm{id}-\partial \partial^{-}\right)$, we obtain $0=\partial^{-} g_{X}\left(\mathrm{id}-\partial \partial^{-}\right)=\partial^{-} g_{X}-\partial^{-} g_{X} \partial \partial^{-}=\partial^{-} g_{X}-$ $g_{S} \partial^{-}$. Hence $\partial^{-} g_{X}=g_{S} \partial^{-}$and the theorem is proved.

The tactical decomposition generated by an automorphism $g=\left(g_{S}, g_{X}\right)$ are the orbits of $g$ on points and blocks. If $R S(g)$ and $R X(g)$ denote the corresponding partition spaces, then $R S(g)=\left\langle\Sigma_{i} g_{S}^{i}(s) \mid s \in S\right\rangle$ and $R X(g)=$ $\left\langle\Sigma_{j} g_{X}^{j}(x) \mid x \in X\right\rangle$. One verifies quite easily that these spaces are characterized by $R S(g)=\left\{v \mid v \in R S, g_{S}(v)=v\right\}=\operatorname{kern}\left(g_{S}-1\right)$ and $R X(g)=\{w \mid w \in R X$, $\left.g_{X}(w)=w\right\}=\operatorname{kern}\left(g_{X}-1\right)$. If rank $I$ is equal to $|S|$, Theorem 3.1 implies $\partial^{-}(R X(g))=R S(g)$. This relation determines the point orbits of $g$. The total number of orbits on points $n\left(g_{S}\right)$ is given by $n\left(g_{S}\right)=n\left(g_{X}\right)-\operatorname{dim}\left(\operatorname{kern}\left(g_{X}-1\right) \cap\right.$ kern $\partial^{-}$). Independently of Theorem 3.1 we calculate the number of orbits of given length.

THEOREM 4.2. Let $g=\left(g_{S}, g_{X}\right)$ be an automorphism of the finite structure $(S, X, \mathrm{I})$ where the rank of $\mathrm{I}$ is $|S|$. Suppose $M=\mathrm{I}^{-} \cdot \mathrm{I}$ for some right inverse $\mathrm{I}^{-}$of I and let $\mu$ be the Möbius function. Then the number $n_{i}\left(g_{S}\right)$ of $g_{S}$-orbits of length $i$ is given by

$$
n_{i}\left(g_{S}\right)=\frac{1}{i} \cdot \sum_{k \mid i} \mu(k) \operatorname{trace}\left(M \cdot g_{X}^{i / k}\right) .
$$

The total orbit numbers $n\left(g_{S}\right)$ and $n\left(g_{X}\right)$ are related through $n\left(g_{X}\right)-n\left(g_{S}\right)=$ $\operatorname{dim}\left(\operatorname{kern}\left(g_{X}-1\right) \cap \operatorname{kern} \partial^{-}\right)=\Sigma_{i \leqslant|X|} \Sigma_{k \mid i} \mu(k) / i$ trace $\left((1-M) \cdot g_{X}^{i / k}\right)$.

Proof. The trace of a permutation matrix $G$ is the number $n_{1}(G)$ of symbols fixed by $G$. The symbols invariant under $G^{2}$ either are points fixed by $G$ or belong to 2-cycles of $G$. Hence $\operatorname{trace}\left(G^{2}\right)=n_{1}(G)+2 n_{2}(G)$ and by induction one proves easily that trace $\left(G^{k}\right)=\Sigma_{i \mid k} i \cdot n_{i}(G)$. Using Möbius inversion, the cycle numbers therefore are given by

$$
n_{i}(G)=\frac{1}{i} \sum_{k \mid i} \mu(k) \operatorname{trace}\left(G^{i / k}\right) .
$$

From $\mathrm{I} g_{X}=g_{S} \mathrm{I}$ and $g_{S}=\mathrm{I} g_{X} \mathrm{I}^{-}$we conclude $g_{S}^{i}=\mathrm{I} g_{X}^{i} \mathrm{I}^{-}$. Therefore,

$$
\begin{aligned}
\operatorname{trace}\left(g_{S}^{i}\right) & =\sum_{r, s, t}(\mathrm{I})_{r s}\left(g_{X}^{i}\right)_{s t}\left(\mathrm{I}^{-}\right)_{t r}=\sum_{r, s, t}\left(\mathrm{I}^{-}\right)_{t r}(\mathrm{I})_{r s}\left(g_{X}^{i}\right)_{s t} \\
& =\operatorname{trace}\left(\left(\mathrm{I}^{-} \cdot \mathrm{I}\right) g_{X}^{i}\right)=\operatorname{trace}\left(M \cdot g_{X}^{i}\right) .
\end{aligned}
$$


Hence we obtain $n_{i}\left(g_{S}\right)=(1 / i) \Sigma_{k \mid i} \mu(k) \operatorname{trace}\left(M \cdot g_{X}^{i / k}\right)$ by the above formula. This proves the first part of Theorem 4.2.

The total orbit number of a permutation $G$ is given by

$$
n(G)=\sum_{i} n_{i}(G)=\sum_{i} \sum_{k \mid i} \frac{\mu(k)}{i} \operatorname{trace}\left(G^{i / k}\right) .
$$

Therefore,

$$
\begin{aligned}
n\left(g_{X}\right)-n\left(g_{S}\right) & =\sum_{i} n_{i}\left(g_{X}\right)-n_{i}\left(g_{S}\right) \\
& =\sum_{i \leqslant|X| k \mid i} \sum_{i} \frac{\mu(k)}{i}\left(\operatorname{trace}\left(g_{X}^{i / k}\right)-\operatorname{trace}\left(g_{S}^{i / k}\right)\right) \\
& =\sum_{i \leqslant|X| k \mid i} \sum_{i} \frac{\mu(k)}{i}\left(\operatorname{trace}\left(g_{X}^{i / k}\right)-\operatorname{trace}\left(M \cdot g_{X}^{i / k}\right)\right) \\
& =\sum_{i \leqslant|X| k \mid i} \sum_{\frac{\mu(k)}{i}} \operatorname{trace}\left((1-M) \cdot g_{x}^{i / k}\right) .
\end{aligned}
$$

The equation $n\left(g_{X}\right)-n\left(g_{S}\right)=\operatorname{dim}\left(\operatorname{kern}\left(g_{X}-1\right) \cap \operatorname{kern} \partial^{-}\right)$follows from Theorem 3.1 as we have pointed out above.

\section{REFERENCES}

1. Block, R. E. : 'Transitive Groups of Collineations on Certain Designs'. Pac. J. Math. 15 (1965), 14-18.

2. Block, R. E. : 'On the Orbits of Collineation Groups'. Math. Z. 69 (1967), 33-49.

3. Cameron, P.: 'Transitivity of Permutation Groups on Unordered Sets'. Math. Z. 148 (1976), 127-139.

4. Cameron, P, and van Lint, J. : Graph theory, coding theory and block designs. LMS Lecture Notes Series 19, Cambridge University Press.

5. Dembowski, P.: 'Verallgemeinerung von Transitivitätsklassen endlicher projektiver Ebenen'. Math. Z. 69 (1958), 59-89.

6. Hering, C.: On codes and projective designs. Kyoto University Mathematics Research Institute, Seminar Notes 344 (1979), 26-60.

7. Hughes, D.: 'Collineations and Generalized Incidence Matrices'. Trans. Amer. Math. Soc. 86 (1957), 284-296.

8. Kantor, W. : 'On Incidence Matrices of Finite Projective and Affine Spaces'. Math. Z. 124 (1972), 315-318.

9. Livingstone, D. and Wagner, A. : 'Transitivity of Finite Permutation Groups on Unordered Sets'. Math. Z. 90 (1965), 393-403.

10. Parker, E. T. : 'On Collineations of Symmetric Designs'. Proc. Amer. Math. Soc. 8 (1957), $350-351$.

11. Rota, G. C. : Finite Operator Calculus. Academic Press, New York, San Francisco, London, 1975.

12. Siemons, I. J.: 'On Partitions and Permutation Groups on Unordered Sets'. Archiv der Mathematik, 38 (1982), 391-403. 
13. Siemons, I. J.: 'Automorphism Groups of Graphs' (to appear in Archiv der Mathematik).

14. Wielandt, H.: Permutation Groups through invariant relations and invariant functions. Lecture Notes, Ohio State University, 1969.

Author's address:

Johannes Siemons,

Department of Mathematics, University College Dublin,

Belfield,

Dublin 4,

Ireland, and

Rittnert Strasse 53

D7500 Karlsruhe

West Germany.

(Received: January 8, 1981) 